\title{
Welcome, Section Editors!
}

\author{
Vojtech Hainer $^{\mathrm{a}}$ Johannes Hebebrand ${ }^{\mathrm{b}}$ \\ ${ }^{a}$ EASO President, Obesity Management Center, Institute of Endocrinology, Prague, Czech Republic \\ ${ }^{b}$ Department of Child and Adolescent Psychiatry and Psychotherapy, University of Duisburg-Essen, Essen, Germany
}

The need for holistic approaches in obesity research and practice was stressed in the Editorial in our first issue of OBESITY FACTS (see also www.karger.com/ofa). Accordingly, we introduce both sections and Section Editors who have individually pledged to commit their expertise to ensure that the review process is both fair and of a high quality. The Executive Committee of the European Association for the Study of Obesity (EASO) decided that the optimal way to achieve its goal of accelerating the high quality review process of submitted papers addressing very different aspects of obesity research is the appointment of Section Editors - each one an expert in a specific field of obesity. Both the existing Editorial Staff and Karger Publishing House strongly endorsed this proposal.

The primary task of the Section Editors is the assignment of good reviewers, capable of prompt and expert constructive review. The team will create a network of reviewers. We deem this networking as extremely important because of the difficulties inherent to finding competent reviewers willing to devote both their knowledge and time to assess the work of submitting authors. High quality reviews are particularly important for junior scientists who tremendously profit from a well written review. In addition, we as readers or as active researchers all profit from well founded reviews. The editors will discuss how to honor this valuable work and how to provide incentives for good reviews.

Other duties of the Section Editors will encompass:

- Invitation of prominent researchers to submit review papers on hot topics or to comment on selected original articles.

- Encouraging exceptional young investigators to submit original articles and intensifying links to The EASO Young Investigators United Network.

- Establishment of contacts to project coordinators, principle investigators and grant recipients of major research projects of the European Union to offer a platform for scientific exchange
- Establishment of contacts to EASO Task Forces with the aim of obtaining updated guidelines for publication in OBEsity FACTS.

- Intensification of contacts to national obesity associations, which can perceive OBESITY FACTS as a platform for publication of representative data on obesity prevalence and lifestyle patterns in addition to articles delineating national prevention strategies.

We have decided to establish five sections and install the following Section Editors:

- Basic Research: Saverio Cinti, Professor of Anatomy, University of Ancona, Italy.

- Epidemiology and Public Health: Thorkild I.A. Sørensen, Professor of Clinical Epidemiology, Institute of Preventive Medicine, Copenhagen University Hospital, Denmark.

- Clinical Aspects and Treatment: Bernhard Ludvik, Professor of Internal Medicine, Department of Internal Medicine III, Division of Endocrinology and Metabolism, University of Vienna, Austria.

- Pediatric Research: Johannes Hebebrand, Professor of Child and Adolescent Psychiatry, Department of Child and Adolescent Psychiatry, University Duisburg-Essen, Germany.

- Bariatric Surgery: Martin Fried, Professor of Surgery, 1st Faculty of Medicine, Charles University, Prague, Czech Republic, who was nominated for this section by the European Chapter of the International Federation for the Surgery of Obesity (IFSO-EC) and approved by EASO.

Our newly appointed Section Editors were asked to outline their plans as pivotal members of the editorial team.

Saverio Cinti encourages submission of original papers as well as reviews on morphology, physiology and pathology of adipose tissues, gastrointestinal tract and central nervous system as well as other relevant organs/tissues. Both studies on humans and animal models are welcome.

\begin{tabular}{ll}
\hline KARGER & @ 2008 S. Karger GmbH, Freiburg \\
Fax +49 761 4520714 & Accessible online at: \\
$\begin{array}{l}\text { E-mail Information@Karger.de } \\
\text { www.karger.com }\end{array}$ & www.karger.com/ofa
\end{tabular}

Prof. Dr. Johannes Hebebrand 
Thorkild Sørensen stresses the necessity to be critical in terms of the evidence base for both observations and interventions in obesity epidemiology and the public health arena. He views this as important because particularly in the latter area there is a tendency to relax more than necessary on the evidence requirements using the arguments that 'it is difficult to conduct the right studies'. He would very much like to support science focusing on translational research aspects with a special emphasis on the output from basic and clinical sciences, not least the genomic work and what emerges from this area in terms of gene-environment interactions, into the public health arena through epidemiology.

Bernhard Ludvik points out that for everybody engaged in the treatment of obesity it is clear that this disorder requires great effort by both the patient and the treating physician. We need to better understand why patients frequently drop out of trials which investigate pharmacological and behavioral interventions including diet and exercise, thus limiting the interpretation of the results regarding general applicability. Our focus must be to encourage well-conducted clinical studies, preferably in multinational settings. In a broader context we need to form partnerships with our patients consistent with their goals and values, we need to stress true cooperation. OBESITY FACTS, as The European Journal of Obesity, should become the first choice for scientists to publish their findings of clinical studies in the field of obesity and related disorders in addition to results that stem from other European research projects.

Johannes Hebebrand perceives the need to focus on genetic and developmental aspects in obesity-related research. Prevalence rates for childhood and adolescent obesity have increased steeply over the past 30 years in many different countries. Many of such children remain obese throughout their lives with dire implications for their health and psychosocial well-being. Measures must be taken to reduce levels of stigmatization which remain high despite recent genetic findings and the unravelling of the physiological mechanisms involved in body weight regulation. Physicians and other therapists need to be aware of the special needs of these children; they must have a solid knowledge as to how to diagnose and treat obesity-related disorders.
Martin Fried believes that the Section Bariatric Surgery and the papers published on this topic will contribute to enhance multidisciplinary understanding of various treatment modalities of obesity, including the possibility of different surgical interventions in individuals with morbid obesity. One of the major treatment-related issues has been that interventions have not been proven to indeed reduce mortality. The year 2007 marks a turning point as two large and long-termed studies revealed that mortality rates of extremely obese individuals are reduced after surgical interventions; despite the fact that these results for obvious reasons were not based on randomized controlled studies, they are certainly promising. Bariatric surgery will provide more detailed insight on physiology of surgical procedures in obese patients from both the purely weight loss perspective as well as from the point of view of "metabolic surgery" with effects on several metabolic aspects of obesity including type 2 diabetes mellitus and other obesity-related co-morbidities. Finally, a fruitful area of investigation has emerged: eating behavior and its underlying mechanisms are now being studied intensively in relation to various operations.

The scientific editors will be vigilant in identifying new subsections in this rapidly evolving field. We believe that the appointment of topic-focused Section Editors will fulfill our expectations and will lead step by step to the high impact of OBESITY FACTS as the official European Journal of Obesity. However, this goal cannot be achieved without close collaboration with you, scientists and clinicians engaged in obesity research across Europe. We look forward to an active collaboration with our authors and readers - the most valuable assets of a scientific journal!

\section{Acknowledgement}

We thank John Kral (Brooklyn, NY, USA) for valuable comments. 


\section{On the Contents of This Issue}

One obviously interesting aspect in dealing with body fat pertains to the importance of gender. Jean Vague in 1956 put it like this: 'In studying the distribution of subcutaneous fat tissue in healthy individuals, one notices considerable differences between the male and female by simply pinching the fat masses with calipers' [1]. In today's world we no longer need to restrict our observations to caliper measurements; we have an arsenal of different anthropometric and clinical measures, imaging techniques, endocrinologic and molecular tools to describe differences between males and females.

Nevertheless, as reported by See Kwok et al. [2] in this fourth edition of OBEsITY FACTS, there is no reason for us to twiddle our thumbs. For one, the majority of research efforts pertaining to an abdominal visceral (android) fat distribution pattern and the metabolic syndrome has focussed on males. Second, cardiovascular disease (CVD) is the largest cause of death in females worldwide. Third, despite the less frequent occurrence of CVD in young females, they catch up as they grow older; due to their higher life expectancy the total numbers of females with CVD actually exceeds that of males. Aware of these facts, Kwok et al. examined the relationship of different indices of fat distribution with cardiovascular risk factors in 13,389 British female department store employees aged 30-65 years. Risk factors increased with age; upon adjustment for age, BMI was most closely correlated with blood pressure. However, the waist for height ratio proved to be more closely correlated with the other risk factors. The authors conclude that hypertension (in females) might more directly reflect the effect of general obesity than of central obesity. Furthermore, they argue that, in comparison with BMI, waist circumference or waist to hip ratio, the waist for height ratio is superior for predicting other CVD risk factors apart from hypertension. Finally, in light of the well known differences in height between Asian females as compared to females of African or European descent the waist for height ratio could prove to be a truly global index and superior to the commonly used waist circumference in assessment of the metabolic syndrome.

The metabolic syndrome can as we all know entail a shortened life span. But does the adipose tissue in itself affect life span and if so by what mechanisms? Matthias Blüher [3] provides a thorough review of data suggesting that the adipose tissue is a pivotal organ in the aging process. He starts off by pointing out that caloric restriction potently extends the life span of several organisms, including mammals. However, the contribution of the reduced adipose tissue mass to this effect is unclear. Indeed, most mouse models of lipoatrophy actually die shortly after birth or have a shortened life span indicating that adipose tissue is not dispensable but instead has an important role such as in maintaining whole body glucose homeostasis, lipid metabolism, and insulin sensitivity. Across different organisms, reduction in adipose tissue mass, either caused by i) caloric restriction, ii) naturally occurring mutations in the insulin/IGF-1 signalling pathway, or iii) in trans- genic animal models, increases life span. The insulin signalling pathway in adipose tissue seems to play a special role with respect to longevity. Accordingly, a prominent characteristic of a centenarian is increased insulin sensitivity compared with younger individuals. Blüher also summarizes the controversial findings on the effects of weight loss on mortality; he argues that loss of fat mass is presumably linked to reduced mortality rates, whereas a reduction of fat-free mass entails elevated risks.

Adipogenic differentiation and the endocrine and metabolic function of adipocytes have mostly been studied in preadipocyte clonal cell lines of murine origin, which are all aneuploid. Only a few human preadipocyte cell lines are available; these are diploid but usually have a short life span. In addition, some were transformed from adipose tissue tumors. Pamela Fischer-Posovsky et al. [4] illustrate the merits of the human Simpson-Golabi-Behmel preadipocyte cell strain, which was first introduced by Wabitsch et al. [5] in 2001 and has subsequently been investigated thoroughly both in the investigators' lab in Ulm and in labs of collaborating scientists. The cells originated from a patient with the respective X-linked overgrowth syndrome. Importantly, the cells retain their capacity to differentiate for up to 50 generations. They make an excellent tool for studying adipocyte biology.

Basak Cinar and Heikki Murtomaa [6] provide the first report in OBESITY FACTS on the association of obesity with decayed, missing and filled teeth (DMFT). The authors compared the results of cross-sectional studies performed on pre-adolescents in Finland and Turkey. To assess DMFT, the 10- to 12-year-olds were subjected to a dental examination, furthermore BMI, leisure time activities, and lifestyle factors were analyzed. The Turkish children had higher rates of obesity and DMFT, with factor analysis revealing that both conditions shared the same cluster of variables including fizzy drinks and prolonged TV consumption. The authors argue that a pediatric dental examination should include assessment of BMI, leisure time activities, dietary habits and socioeconomic status.

\section{References}

1 Vague J: The degree of masculine differentiation of obesities. Am J Clin Nutr 1956;4:20-34.

2 Kwok S, McElduff P, Ashton DW, Lowe GDO, Wood D, Humphries SE, Charlton-Menys V, Durrington PN: Indices of obesity and cardiovascular risk factors in British women. Obesity Facts 2008;1(4):190-195.

3 Blüher M: Fat Tissue and Long Life. Obesity Facts 2008;1(4):176-182.

4 Fischer-Posovszky P, Newell FS, Wabitsch M, Tornqvist HE: Human SGBS cells - a unique tool for studies of human fat cell biology. Obesity Facts 2008;1(4):184-189.

5 Wabitsch M, Brenner RE, Melzner I, Braun M, Moller P, Heinze E, Debatin KM, Hauner H: Characterization of a human preadipocyte cell strain with high capacity for adipose differentiation. Int J Obes Relat Metab Disord 2001;25:8-15.

6 Cinar AB, Murtomaa $\mathrm{H}$ : Clustering of obesity and dental health with lifestyle factors among turkish and finnish pre-adolescents. Obesity Facts 2008;1(4): 196-202. 